\title{
On Abelian and Related Fuzzy Subsets of Groupoids
}

\author{
Seung Joon Shin, ${ }^{1}$ Hee Sik Kim, ${ }^{2}$ and J. Neggers ${ }^{3}$ \\ ${ }^{1}$ Department of Physics, University of Michigan, Ann Arbor, MI 48109, USA \\ ${ }^{2}$ Department of Mathematics, Research Institute for Natural Sciences, Hanyang University, Seoul 133-791, Republic of Korea \\ ${ }^{3}$ Department of Mathematics, University of Alabama, Tuscaloosa, AL 35487-0350, USA
}

Correspondence should be addressed to Hee Sik Kim; heekim@hanyang.ac.kr

Received 12 August 2013; Accepted 15 September 2013

Academic Editors: M. Aiguier and M. I. Ali

Copyright (C) 2013 Seung Joon Shin et al. This is an open access article distributed under the Creative Commons Attribution License, which permits unrestricted use, distribution, and reproduction in any medium, provided the original work is properly cited.

We introduce the notion of abelian fuzzy subsets on a groupoid, and we observe a variety of consequences which follow. New notions include, among others, diagonal symmetric relations, several types of quasi orders, convex sets, and fuzzy centers, some of whose properties are also investigated.

\section{Introduction}

The notion of a fuzzy subset of a set was introduced by Zadeh [1]. His seminal paper in 1965 has opened up new insights and applications in a wide range of scientific fields. Rosenfeld [2] used the notion of a fuzzy subset to set down corner stone papers in several areas of mathematics. Mordeson and Malik [3] published a remarkable book, Fuzzy commutative algebra, presented a fuzzy ideal theory of commutative rings, and applied the results to the solution of fuzzy intersection equations. The book included all the important work that has been done on $L$-subspaces of a vector space and on $L$ subfields of a field.

Kim and Neggers [4] introduced the notion of $\operatorname{Bin}(X)$ and obtained a semigroup structure. Fayoumi [5] introduced the notion of the center $Z \operatorname{Bin}(X)$ in the semigroup $\operatorname{Bin}(X)$ of all binary systems on a set $X$ and showed that aroupoid $(X, \bullet) \epsilon$ $Z \operatorname{Bin}(X)$ if and only if it is a locally zero groupoid.

In this paper, we introduce the notion of abelian fuzzy subgroupoids on a groupoid and discuss diagonal symmetric relations, convex sets, and fuzzy centers on $\operatorname{Bin}(X)$.

\section{Preliminaries}

Given a nonempty set $X$, we let $\operatorname{Bin}(X)$ denote the collection of all groupoids $(X, *)$, where $*: X \times X \rightarrow X$ is a map and $*(x, y)$ is written in the usual product form. Given elements
$(X, *)$ and $(X, \bullet)$ of $\operatorname{Bin}(X)$, define a product “ $\square$ " on these groupoids as follows:

$$
(X, *) \square(X, \bullet)=(X, \square)
$$

where

$$
x \square y=(x * y) \cdot(y * x)
$$

for any $x, y \in X$. Using that notion, Kim and Neggers proved the following theorem.

Theorem 1 (see [4]). (Bin $(X), \square)$ is a semigroup; that is, the operation " $\square$ " as defined in general is associative. Furthermore, the left-zero-semigroup is the identity for this operation.

Let $Z \operatorname{Bin}(X)$ denote the collection of elements $(X, \bullet)$ of $\operatorname{Bin}(X)$ such that $(X, *) \square(X, \bullet)=(X, \bullet) \square(X, *)$, for all $(X, *) \in \operatorname{Bin}(X)$; that is, $Z \operatorname{Bin}(X)=\{(X, \bullet) \in \operatorname{Bin}(X)$ $(X, *) \square(X, \bullet)=(X, \bullet) \square(X, *)$, for all $(X, *) \in \operatorname{Bin}(X)\}$. We call $Z \operatorname{Bin}(X)$ the center of the semigroup $\operatorname{Bin}(X)$.

Proposition 2 (see [5]). If $(X, \bullet) \in Z \operatorname{Bin}(X)$, then $x \bullet x=x$ for all $x \in X$.

Proposition 3 (see [5]). Let $(X, \bullet) \in Z B \operatorname{Bin}(X)$. If $x \neq y$ in $X$, then $(\{x, y\}, \bullet)$ is either a left-zero-semigroup or a right-zerosemigroup. 


\section{Abelian Fuzzy Groupoids}

Let $(X, *) \in \operatorname{Bin}(X)$. A map $\mu: X \rightarrow[0,1]$ is said to be abelian fuzzy if $\mu(x * y)=\mu(y * x)$ for all $x, y \in X$.

Example 4. Let $(X, *)$ be a left-zero-semigroup; that is, $x * y=$ $x$ for all $x, y \in X$. Let $\mu: X \rightarrow[0,1]$ be an abelian fuzzy subset of $X$. Then, $\mu(x)=\mu(x * y)=\mu(y * x)=\mu(y)$ for all $x, y \in X$. It follows that $\mu$ is a constant map.

Similarly, every abelian fuzzy subset of a right-zerosemigroup is also a constant function.

Proposition 5. If $(X, *) \in Z \operatorname{Bin}(X)$, then every abelian fuzzy subset on $(X, *)$ is a constant function.

Proof. Assume that $\mu$ is an abelian fuzzy subset of $(X, *)$. Then, $\mu(x * y)=\mu(y * x)$ for all $x, y \in X$. Let $(X, *) \in$ $Z \operatorname{Bin}(X)$. By Proposition 3, if $x \neq y$ in $X$, then $(\{x, y\}, *)$ is either a left-zero-semigroup or a right-zero-semigroup. It follows that either $x * y=x, y * x=y$ or $x * y=y, y * x=x$. In either cases, we obtain $\mu(x)=\mu(y)$ for all $x, y \in X$, proving that $\mu$ is a constant function.

Given a groupoid $(X, *) \in \operatorname{Bin}(X)$, we denote the set of all abelian fuzzy subgroupoids on $(X, *)$ by $A(X, *)$.

Proposition 6. Let $(X, *) \in \operatorname{Bin}(X)$. Then, $(X, *)$ is commutative if and only if $A(X, *)=[0,1]^{X}$.

Proof. Assume that $(X, *)$ is not commutative; that is, there exist $x, y \in X$ such that $x * y \neq y * x$. If we let $\alpha:=x * y$ and let $\mu:=\chi_{\alpha}$ be the characteristic function of $\alpha$, then $\mu(x * y)=1$, $\mu(y * x)=0$, proving that $\mu$ is not an abelian fuzzy subset of $(X, *)$. The converse is straightforward.

Given $(X, *) \in \operatorname{Bin}(X)$, we define a fuzzy subset $\mu_{\alpha}: X \rightarrow$ $[0,1]$ by $\mu_{\alpha}(x):=\alpha$ for all $x \in X$, where $\alpha \in[0,1]$. Denote by $C(X, *):=\left\{\mu_{\alpha} \mid \alpha \in[0,1]\right\}$. Then, $C(X, *) \subseteq A(X, *)$ for all groupoids $(X, *)$ whatsoever. Thus, the extreme of noncommutativity is the situation $C(X, *)=A(X, *)$.

Proposition 7. Let $\mu \in A(X, *)$. If $\mu$ is one-one, then $(X, *)$ is commutative.

Proof. If $\mu \in A(X, *)$, then $\mu(x * y)=\mu(y * x)$ for all $x, y \in X$. Since $\mu$ is one-one, we have $x * y=y * x$ for all $x, y \in X$.

Given $(X, *) \in \operatorname{Bin}(X)$, we define a set $(X, *)_{a}:=\{(x, y) \in$ $X \times X \mid x * y=y * x\}$. Note that $(x, y) \in(X, *)_{a}$ implies $(y, x) \in(X, *)_{a}$ as well. If we let $\nu:=\chi_{(X, *)_{a}}$ be the characteristic function of $(X, *)_{a}$, then $v$ is an abelian fuzzy subgroupoid on $X \times X$.

Proposition 8. Let $\mu: X \rightarrow[0,1]$ be a fuzzy subset of $X$. If we define $(X, *)_{a}^{\mu}:=\{(x, y) \in X \times X \mid \mu(x * y)=\mu(y * x)\}$, then

(i) $(X, *)_{a} \subseteq(X, *)_{a}^{\mu}$, (ii) if $\mu$ is one-one, then $(X, *)_{a}=(X, *)_{a}^{\mu}$,

(iii) if $\mu$ is constant, then $(X, *)_{a}^{\mu}=X \times X$.

Proof. It is straightforward.

Theorem 9. If $(X, *) \in \operatorname{Bin}(X)$, then there exists a fuzzy subset $\mu$ of $X$ such that $(X, *)_{a}=(X, *)_{a}^{\mu}$.

Proof. Assume that there exists $(X, *) \in \operatorname{Bin}(X)$ such that $(X, *)_{a} \neq(X, *)_{a}^{\mu}$ for any fuzzy subset $\mu$ of $X$. Then, there exists an element $(x, y) \in(X, *)_{a}^{\mu} \backslash(X, *)_{a}$. It follows that $\mu(x * y)=\mu(y * x)$, but $x * y \neq y * x$ for some $x, y \in X$. If we let $\alpha:=x * y$ and let $\mu:=\chi_{\alpha}$ be the characteristic function of $\alpha$, then $\mu(x * y)=1$, but $\mu(y * x)=0$, which proves that $(x, y) \notin(X, *)_{a}^{\mu}$.

Proposition 10. Let $(X, *) \in \operatorname{Bin}(X)$ and let $\cdot$ be the usual product on the set of real numbers. If $\mu:(X, *) \rightarrow([0,1], \cdot)$ is a homomorphism, then $\mu \in A(X, *)$ and $(X, *)_{a}^{\mu}=X \times X$.

Proof. If $\mu:(X, *) \rightarrow([0,1], \cdot)$ is a homomorphism, then $\mu(x * y)=\mu(x) \cdot \mu(y)=\mu(y) \cdot \mu(x)=\mu(y * x)$ for all $x, y \in X$; that is, $\mu \in A(X, *)$.

If $(x, y) \in X \times X$, then $\mu(x * y)=\mu(x) \cdot \mu(y)=\mu(y * x)$, proving that $(x, y) \in(X, *)_{a}^{\mu}$.

Example 11. Let $(X, *) \in \operatorname{Bin}(X)$. Define a binary operation “*” on $[0, \infty)$ by $x \star y:=(1 / 2)(x+y)$ for all $x, y \in[0, \infty)$. If $\mu:(X, *) \rightarrow([0, \infty), \star)$ is a homomorphism, then $\mu(x *$ $y)=\mu(x) \star \mu(y)=(1 / 2)(x+y)=\mu(y * x)$ for all $x, y \in X$, which proves that $\mu \in A(X, *)$ and $(X, *)_{a}^{\mu}=X \times X$.

\section{Diagonal Symmetric Relations}

Given $(X, *) \in \operatorname{Bin}(X)$, we denote $\triangle(X):=\{(x, x) \mid x \in X\}$. Then, $\triangle(X) \subseteq(X, *)_{a}$. In particular, if $(X, *)$ is a left-zerosemigroup, then $\triangle(X)=(X, *)_{a}$.

Let $X:=\mathbf{R}$ be the set of all real numbers, and let "-" be the usual subtraction on $X$. Then, $(X,-)_{a}=\{(x, y) \mid x-y=$ $y-x\}=\{(x, y) \mid x=y\}=\triangle(X)$.

Let $X:=\mathbf{R}$ be the set of all real numbers, and let $(X, *, f)$ be a leftoid; that is, $x * y:=f(x)$ for all $x, y \in X$, where $f$ : $\mathbf{R} \rightarrow \mathbf{R}$ is an even function. If we denote $\triangle^{\prime}(X):=\{(x,-x) \mid$ $x \in X\}$, then $x *(-x)=f(x)=f(-x)=-x * x$ for all $x \in X$. It follows that $\triangle^{\prime}(X) \subseteq(X, *, f)$.

Let $X$ be a nonempty set, and let $S \subseteq X \times X$ such that $\triangle(X) \subseteq S$. $S$ is said to be diagonal symmetric if $(x, y) \in S$, then $(y, x) \in S$ as well. If $(X, *) \in \operatorname{Bin}(X)$, then $(X, *)_{a}$ is diagonal symmetric. Define a map $\Phi_{a}: \operatorname{Bin}(X) \rightarrow P(X \times X)$ by $\Phi_{a}(X, *):=(X, *)_{a}$.

Proposition 12. If $S$ is a diagonal symmetric relation on $X$, then there exists $(X, *) \in \operatorname{Bin}(X)$ such that $\Phi_{a}(X, *)=S$.

Proof. Let $S$ be a diagonal symmetric relation on $X$. Define a binary operation “*” on $X$ by

$$
x * y:= \begin{cases}x & \text { if }(x, y) \notin S \\ z & \text { otherwise }\end{cases}
$$


where $z$ is an element of $X$ satisfying $z=x * y=y * x$. Then, $(X, *)_{a}=S$. In fact, if $(x, y) \in S$, then $x * y=y * x$, and hence, $(x, y) \in(X, *)_{a}$. Assume that $(x, y) \in(X, *)_{a} \backslash$ $S$. Then, since $S$ is symmetric diagonal, we have $x * y=x$ and $(y, x) \notin S$. It follows that $x * y=x, y * x=y$. Since $(x, y) \in(X, *)_{a}$, we have $x=x * y=y * x=y$, proving that $(x, y)=(x, x) \in \triangle(X) \subseteq S$, a contradiction. Hence, $\Phi_{a}(X, *)=(X, *)_{a}=S$.

If $S$ and $T$ are diagonal symmetric relations on $X$, then the same is true for $S \cap T$ and for $S \cup T$, while $\triangle(X)$ itself is also a diagonal symmetric relation on $X$. In the latter case, the left-zero-semigroup is among the possible groupoids for which $\Phi_{a}(X, *)=(X, *)_{a}=\triangle(X)$.

Proposition 13. Let $(X, *),(X, \bullet) \in \operatorname{Bin}(X)$. If $(X, \square)=$ $(X, *) \square(X, \bullet)$, then $(X, *)_{a} \subseteq(X, \square)_{a}$.

Proof. If $(x, y) \in(X, *)_{a}$, then $x * y=y * x$, and hence, $x \square y=(x * y) \cdot(y * x)=(y * x) \square(x * y)=y \square x$. Hence, $(x, y) \in(X, \square)_{a}$.

Thus, if $(X, *)$ and $(X, \square)$ are given and the question comes up whether $(X, \square)=(X, *) \square(X, \bullet)$ for some $(X, \bullet)$, then $(X, *)_{a} \subseteq(X, \square)_{a}$ is a necessary precondition for this to be the case. For example, if $\triangle(X)=(X, \square)_{a}$, then $(X, *)_{a}=$ $\triangle(X)$ as well. Thus, we find that in $(\operatorname{Bin}(X), \square)$, "the product does not decrease commutativity" as a general principle.

Proposition 14. Let $(X, *) \in \operatorname{Bin}(X)$. If $(A, *)$ is a subgroupoid of $(X, *)$, then $(X, *)_{a} \cap(A \times A)=(A, *)_{a}$.

Proof. The proof is straightforward.

Proposition 15. Let $(X, *),(X, \bullet) \in \operatorname{Bin}(X)$. If $(X, \square)=$ $(X, *) \square(X, \bullet)$, then $A(X, \bullet) \subseteq A(X, \square)$.

Proof. If $\mu \in A(X, \bullet)$, then $\mu(x \bullet y)=\mu(y \bullet x)$ for all $x, y \in X$. It follows that

$$
\begin{aligned}
\mu(x \square y) & =\mu((x * y) \bullet(y * x)) \\
& =\mu((y * x) \bullet(x * y))=\mu(y \square x) .
\end{aligned}
$$

Hence, $\mu \in A(X, \square)$.

Proposition 16. Let $(X, *) \in \operatorname{Bin}(X)$ satisfy the condition: for any $a, b \in X$, there exist $x, y \in X$ such that $a=x * y, b=y * x$. If $(X, \square)=(X, *) \square(X, \bullet)$, then $A(X, \bullet)=A(X, \square)$.

Proof. If $\mu \in A(X, \square)$, then $\mu(x \square y)=\mu(y \square x)$ for all $x, y \in$ $X$. Given $a, b \in X$, by assumption, we have $x, y \in X$ such that $a=x * y, b=y * x$. It follows that

$$
\begin{aligned}
\mu(a \bullet b) & =\mu((x * y) \bullet(y * x)) \\
& =\mu(x \square y)=\mu(y \square x) \\
& =\mu((y * x) \bullet(x * y))=\mu(b \cdot a) .
\end{aligned}
$$

Hence, $\mu \in A(X, \bullet)$; that is, $A(X, \square) \subseteq A(X, \bullet)$. By Proposition 15, we prove the proposition.
Let $(X, *),(X, \bullet) \in \operatorname{Bin}(X)$. Define a relation " $\leq$ " on $\operatorname{Bin}(X)$ by $(X, *) \leq(X, \bullet)$ if and only if $(X, *)_{a}^{\mu} \subseteq(X, \bullet)_{a}^{\mu}$ for any fuzzy subset $\mu: X \rightarrow[0,1]$.

Proposition 17. The relation $\leq$ is a quasi order on $\operatorname{Bin}(X)$.

Proof. Since $(X, *)_{a}^{\mu} \subseteq(X, *)_{a}^{\mu}$ for any fuzzy subset $\mu: X \rightarrow$ $[0,1]$, we have $(X, *) \leq(X, *)$ for all $(X, *) \in \operatorname{Bin}(X)$.

If $(X, *) \leq(X, \bullet)$ and $(X, \bullet) \leq(X, \star)$, then $(X, *)_{a}^{\mu} \subseteq$ $(X, \bullet)_{a}^{\mu}$ and $(X, \bullet)_{a}^{\mu} \subseteq(X, \star)_{a}^{\mu}$, and hence, $(X, *)_{a}^{\mu} \subseteq(X, \star)_{a}^{\mu}$, for any fuzzy subset $\mu: X \rightarrow[0,1]$. It follows that $(X, *) \leq$ $(X, \star)$.

Note that the relation $\leq$ described in Proposition 17 need not be a partial order on $\operatorname{Bin}(X)$, since $(X, *)_{a}^{\mu}=(X, \bullet)_{a}^{\mu}$ for any fuzzy subset $\mu: X \rightarrow[0,1]$ does not imply $(X, *)=$ $(X, \bullet)$

Given $(X, *) \in \operatorname{Bin}(X)$, we define

$$
\begin{aligned}
{[(X, *)]:=\left\{(X, \bullet) \in \operatorname{Bin}(X) \mid(X, *)_{a}^{\mu}\right.} \\
\left.\quad=(X, \bullet)_{a}^{\mu}, \quad \forall \mu \text { : fuzzy subset of } X\right\} .
\end{aligned}
$$

Let $\langle\operatorname{Bin}(X)\rangle:=\{[(X, *)] \mid(X, *) \in \operatorname{Bin}(X)\}$. If we define a relation $\leq_{q}$ on $\langle\operatorname{Bin}(X)\rangle$ by

$$
[(X, *)] \leq_{q}[(X, \bullet)] \Longleftrightarrow(X, *) \leq(X, \bullet) .
$$

then it is easy to see that $\left(\left\langle\operatorname{Bin}(X), \leq_{q}\right\rangle\right)$ is a partially ordered set. For partially ordered sets, we refer to [6].

Proposition 18. If $[(X, *)]=[(X, \bullet)]$ in $\langle\operatorname{Bin}(X)\rangle$, then $A(X, *)=A(X, \bullet)$.

Proof. Assume that $[(X, *)]=[(X, \bullet)]$. Then, $(X, *)_{a}^{\mu}=$ $(X, \bullet)_{a}^{\mu}$ for any fuzzy subset $\mu: X \rightarrow[0,1]$. It follows that

$$
\begin{aligned}
\mu \in A(X, *) & \Longleftrightarrow \mu(x * y) \\
& =\mu(y * x) \quad \forall x, y \in X \\
& \Longleftrightarrow(X, *)_{a}^{\mu}=X \times X=(X, \bullet)_{a}^{\mu} \\
& \Longleftrightarrow \mu(x \bullet y)=\mu(y \bullet x) \quad \forall x, y \in X \\
& \Longleftrightarrow \mu \in A(X, \bullet),
\end{aligned}
$$

proving that $A(X, *)=A(X, \bullet)$.

\section{Convex Sets}

Proposition 19. Let $\mu, \nu$ be fuzzy subsets of $(X, *)$. If $\lambda:=t \mu+$ $(1-t) v$, where $t \in[0,1]$, then $(X, *)_{a}^{\mu} \cap(X, *)_{a}^{\nu} \subseteq(X, *)_{a}^{\lambda}$.

Proof. If $(x, y) \in(X, *)_{a}^{\mu} \cap(X, *)_{a}^{v}$, then $\mu(x * y)=\mu(y * x)$ and $\nu(x * y)=\nu(y * x)$ for all $x, y \in X$. It follows that

$$
\begin{aligned}
\lambda(x * y) & =t \mu(x * y)+(1-t) \nu(x * y) \\
& =t \mu(y * x)+(1-t) \nu(y * x) \\
& =\lambda(y * x),
\end{aligned}
$$

proving that $(x, y) \in(X, *)_{a}^{\lambda}$. 
In Proposition 19, the equality does not hold in general. See the following example.

Example 20. Let $X:=[0,1]$. Define a binary operation “*” on $X$ by $x * y:=x$ for all $x, y \in X$; that is, $(X, *)$ is a left-zero-semigroup. Define two fuzzy subsets $\mu, v$ on $X$ by $\mu(x):=x, \nu(x):=1-x$ for all $x \in X$. Then, $\mu$ and $\nu$ are oneone mappings. Let $\epsilon$ be a real number such that $0<\epsilon<1 / 2$, and let $u:=(1 / 2)-\epsilon, v:=(1 / 2)+\epsilon$. Then, $\mu(v)-\mu(u)=$ $\nu(u)-v(v) \neq 0$. Let $\lambda:=(1 / 2)(\mu+\nu)$. Then,

$$
\begin{aligned}
\lambda(u * v) & -\lambda(v * u) \\
= & \frac{1}{2}[\mu(u * v)+v(u * v)] \\
& -\frac{1}{2}[\mu(v * u)+v(v * u)] \\
= & \frac{1}{2}[\mu(u * v)-\mu(v * u)] \\
& +\frac{1}{2}[v(u * v)-v(v * u)] \\
= & \frac{1}{2}[\mu(u)-\mu(v)] \\
& +\frac{1}{2}[v(u)-v(v)]=0,
\end{aligned}
$$

which shows that $(u, v) \in(X, *)_{a}^{\lambda}$.

Since $(X, *)$ is a left-zero-semigroup and $\mu$ is one-one, we have $(X, *)_{a}^{\mu}=\{(x, y) \mid \mu(x * y)=\mu(y * x)\}=\{(x, y) \mid$ $\mu(x)=\mu(y)\}=\triangle(X)=(X, *)_{a}^{v}$. This shows that $(u, v) \notin$ $\triangle(X)=(X, *)_{a}^{\mu} \cap(X, *)_{a}^{\nu}$.

Corollary 21. Let $\mu, \nu$ be fuzzy subsets of $(X, *)$. If $\lambda:=t \mu+(1-$ $t) v$, where $t \in[0,1]$, and if $(X, *)_{a}^{\mu}=(X, *)_{a}^{\nu}$, then $(X, *)_{a}^{\mu}=$ $(X, *)_{a}^{v} \subseteq(X, *)_{a}^{\lambda}$.

Theorem 22. Let $\mu, v$ be fuzzy subsets of $(X, *)$. If $\lambda_{i}:=t_{i} \mu+$ $\left(1-t_{i}\right) v,(i=1,2)$, where $t_{1}, t_{2} \in[0,1]$ with $t_{1} \neq t_{2}$, then $(X, *)_{a}^{\lambda_{1}} \cap(X, *)_{a}^{\lambda_{2}} \subseteq(X, *)_{a}^{\mu} \cap(X, *)_{a}^{v}$.

Proof. If $(x, y) \in(X, *)_{a}^{\lambda_{1}} \cap(X, *)_{a}^{\lambda_{2}}$, then

$$
\lambda_{i}(x * y)-\lambda_{i}(y * x)=0,
$$

where $i=1,2$. It follows that

$$
\begin{gathered}
t_{1}[\mu(x * y)-\mu(y * x)]+\left(1-t_{1}\right) \\
\times[\nu(x * y)-\nu(y * x)]=0 \\
t_{2}[\mu(x * y)-\mu(y * x)]+\left(1-t_{2}\right) \\
\times[\nu(x * y)-\nu(y * x)]=0 .
\end{gathered}
$$

Since $\left|\begin{array}{ll}t_{1} & 1-t_{1} \\ t_{2} & 1-t_{2}\end{array}\right|=t_{1}\left(1-t_{2}\right)-t_{2}\left(1-t_{1}\right)=t_{1}-t_{2} \neq 0$, by Cramer's rule, we have $\mu(x * y)-\mu(y * x)=\nu(x * y)-\nu(y * x)=0$, so that $(x, y) \in(X, *)_{a}^{\mu} \cap(X, *)_{a}^{v}$. This proves the theorem.
Corollary 23. Let $\mu, \nu$ be fuzzy subsets of $(X, *)$, and let $\lambda_{i}:=$ $t_{i} \mu+\left(1-t_{i}\right) v,(i=1,2)$, where $t_{1}, t_{2} \in[0,1]$ with $t_{1} \neq t_{2}$. If $(X, *)_{a}^{\mu}=(X, *)_{a}^{\nu}$, then $(X, *)_{a}^{\lambda_{1}} \cap(X, *)_{a}^{\lambda_{2}}=(X, *)_{a}^{\mu}=$ $(X, *)_{a}^{v}$.

Proof. It follows immediately from Corollary 21 and Theorem 22.

We give a pause to find some examples of fuzzy subsets $\mu, \nu$ of $X$ and groupoids $(X, *)$ and $(X, \bullet)$ such that

$$
\text { (I) } \quad(X, \bullet)_{a}^{\mu} \subseteq(X, *)_{a}^{\mu}, \quad(X, *)^{\mu} \subseteq(X, \bullet)_{a}^{\nu}
$$

or

$$
\text { (II) } \quad(X, *)_{a}^{\nu} \subseteq(X, *)_{a}^{\mu}, \quad(X, \bullet)^{\mu} \subseteq(X, \bullet)_{a}^{\nu}
$$

or both (I) and (II) simultaneously.

Example 24. Let $X:=\{a, b, c\}$ with the tables:

$$
\begin{array}{l|llll|lll}
* & a & b & c & \bullet & a & b & c \\
\hline a & a & b & c & a & a & b & c \\
b & c & a & b & b & b & b & c \\
c & b & c & a & c & c & c & c
\end{array}
$$

Define two fuzzy subsets $\mu, v$ on $X$ by $\mu(a)=1, \mu(b)=$ $1 / 2, \mu(c)=1 / 3$ and $\nu(a)=1, \nu(b)=\nu(c)=1 / 2$. Then, it is easy to see that $(X, *)_{a}=\{(a, a),(b, b),(c, c)\}=(X, *)_{a}^{\mu}$, $(X, *)_{a}^{v}=(X, \bullet)_{a}^{v}=(X, \bullet)_{a}^{\nu}=X \times X$. Hence, $(X, *)_{a}^{\mu} \subseteq(X, \bullet)_{a}^{\mu}$, $(X, \bullet)_{a}^{v}=(X, *)_{a}^{v}$, and $(X, *)_{a}^{\mu} \subseteq(X, *)_{a}^{v},(X, *)_{a}^{v} \subseteq(X, \bullet)_{a}^{v}$.

\section{Fuzzy Center}

Let $(X, *) \in \operatorname{Bin}(X)$, and let $\mu$ be fuzzy subsets of $X$. Define a set $Z_{\mu}(X, *)$ by

$$
Z_{\mu}(X, *):=\{x \in X \mid \mu(x * y)=\mu(y * x)\} .
$$

We call it a $\mu$-center of $(X, *)$. In Example $24, Z_{\mu}(X, *)=\emptyset$, $Z_{\nu}(X, *)=X$.

Proposition 25. Let $(X, *) \in \operatorname{Bin}(X)$. Then, $x \in Z_{\mu}(X, *)$ if and only if $(\{x\} \times X) \cup(X \times\{x\}) \subseteq(X, *)_{a}^{\mu}$.

Proof. It follows that

$$
\begin{aligned}
x \in Z_{\mu}(X, *) & \Longleftrightarrow \mu(x * y) \\
& =\mu(y * x), \quad \forall y \in X \\
& \Longleftrightarrow(x, y),(y, x) \in(X, *)_{a}^{\mu} \\
& \Longleftrightarrow(\{x\} \times X) \cup(X \times\{x\}) \subseteq(X, *)_{a}^{\mu} .
\end{aligned}
$$

Let $(X, *) \in \operatorname{Bin}(X)$, and let $\mu: X \rightarrow[0,1]$ be a fuzzy subset of $X$. Define a set $O Z_{\mu}(X, *)$ by

$$
\begin{aligned}
\mathrm{OZ}_{\mu}(X, *):=\{x & \in X \mu(x * y) \\
& =\mu(y * x) \Longrightarrow \mu(x)=\mu(y)\} .
\end{aligned}
$$

With the notion of $\mu$-center, we obtain the following. 
Proposition 26. If $O Z_{\mu}(X, *) \cap Z_{\mu}(X, *) \neq \emptyset$, then $\mu$ is a constant function.

Proof. If $x \in O Z_{\mu}(X, *) \cap Z_{\mu}(X, *)$, then $\mu(x * y)=\mu(y * x)$ for all $y \in X$. Since $x \in O Z_{\mu}(X, *)$, we have $\mu(x)=\mu(y)$ for all $y \in X$, proving that $\mu$ is a constant function.

Proposition 27. If $\mu$ is a constant function, then $O Z_{\mu}(X, *)=$ $X=Z_{\mu}(X, *)$.

Proof. $\mu$ is a constant function, then $\mu(x * y)=\mu(y * x)$ for all $x, y \in X$. It follows that $Z_{\mu}(X, *)=X$.

Assume that $x \notin O Z_{\mu}(X, *)$. Then, there exists an $y \in$ $X$ such that $\mu(x * y)=\mu(y * x)$, but $\mu(x) \neq \mu(y)$. Since $\mu$ is a constant function, we obtain $\mu(x)=\mu(y) \neq \mu(x)$, a contradiction. Hence, $O Z_{\mu}(X, *)=X$.

\section{References}

[1] L. A. Zadeh, "Fuzzy sets," Information and Control, vol. 8, no. 3, pp. 338-353, 1965.

[2] A. Rosenfeld, "Fuzzy groups," Journal of Mathematical Analysis and Applications, vol. 35, no. 3, pp. 512-517, 1971.

[3] J. M. Mordeson and D. D. Malik, Fuzzy Commutative Algebra, World Scientific, Singapore, 1998.

[4] H. S. Kim and J. Neggers, "The semigroups of binary systems and some perspectives," Bulletin of the Korean Mathematical Society, vol. 45, no. 4, pp. 651-661, 2008.

[5] H. F. Fayoumi, "Locally-zero groupoids and the center of $\operatorname{Bin}(X)$," Communications of the Korean Mathematical Society, vol. 26, no. 2, pp. 163-168, 2011.

[6] J. Neggers and H. S. Kim, Basic Posets, World Scientific, Singapore, 1998. 


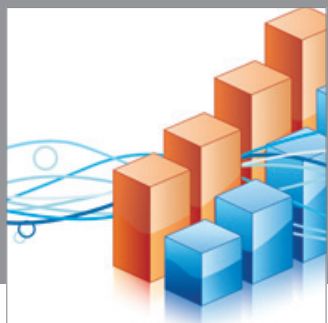

Advances in

Operations Research

mansans

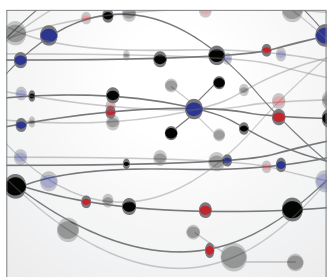

The Scientific World Journal
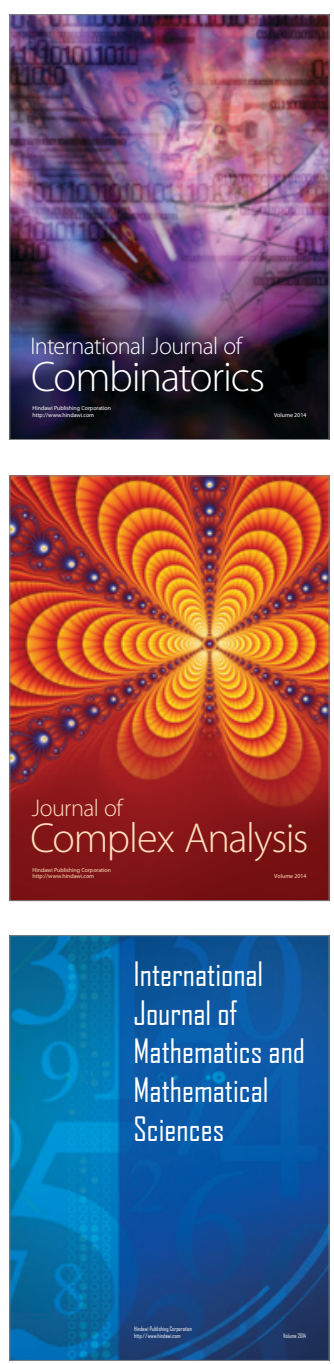
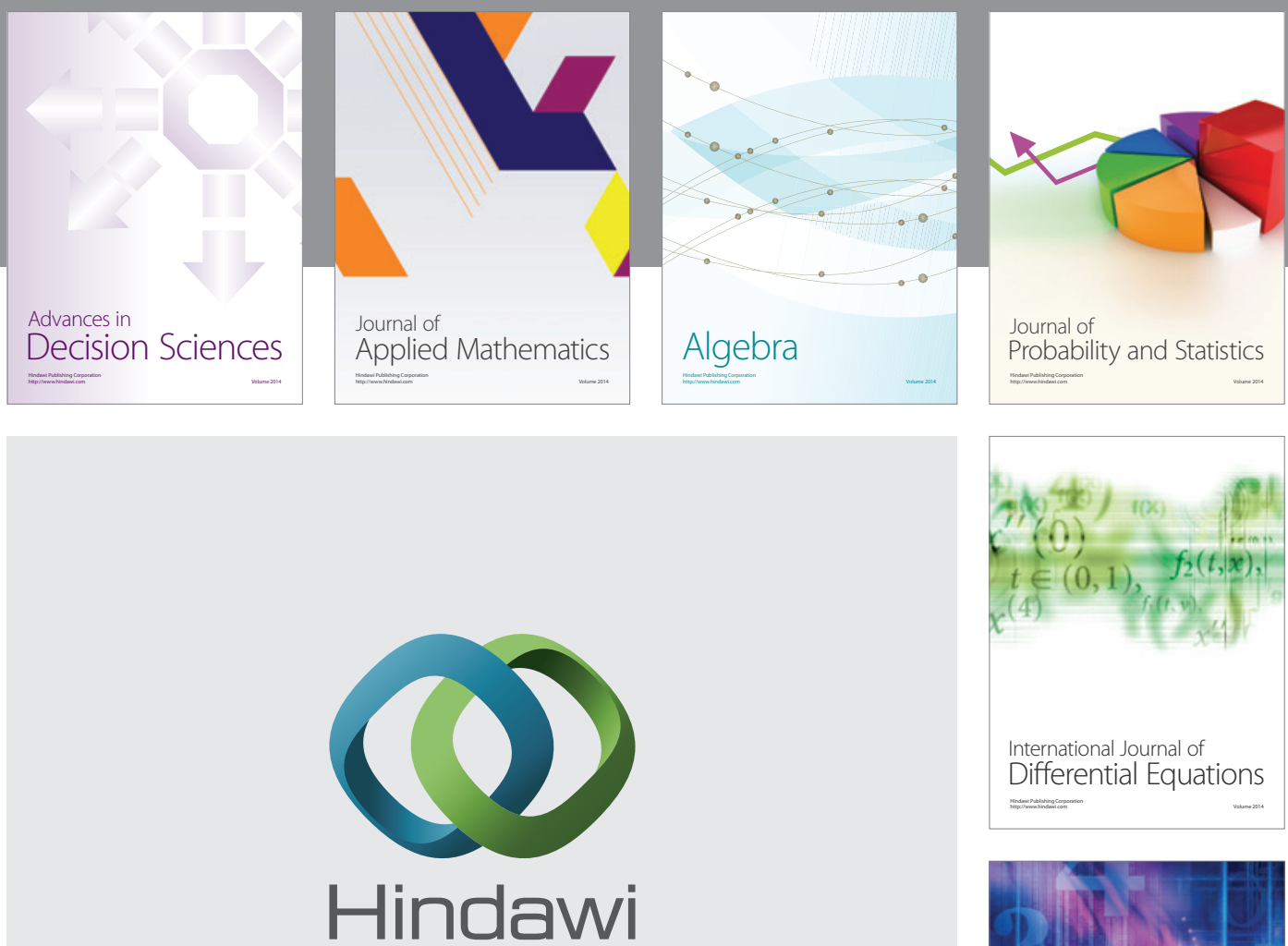

Submit your manuscripts at http://www.hindawi.com
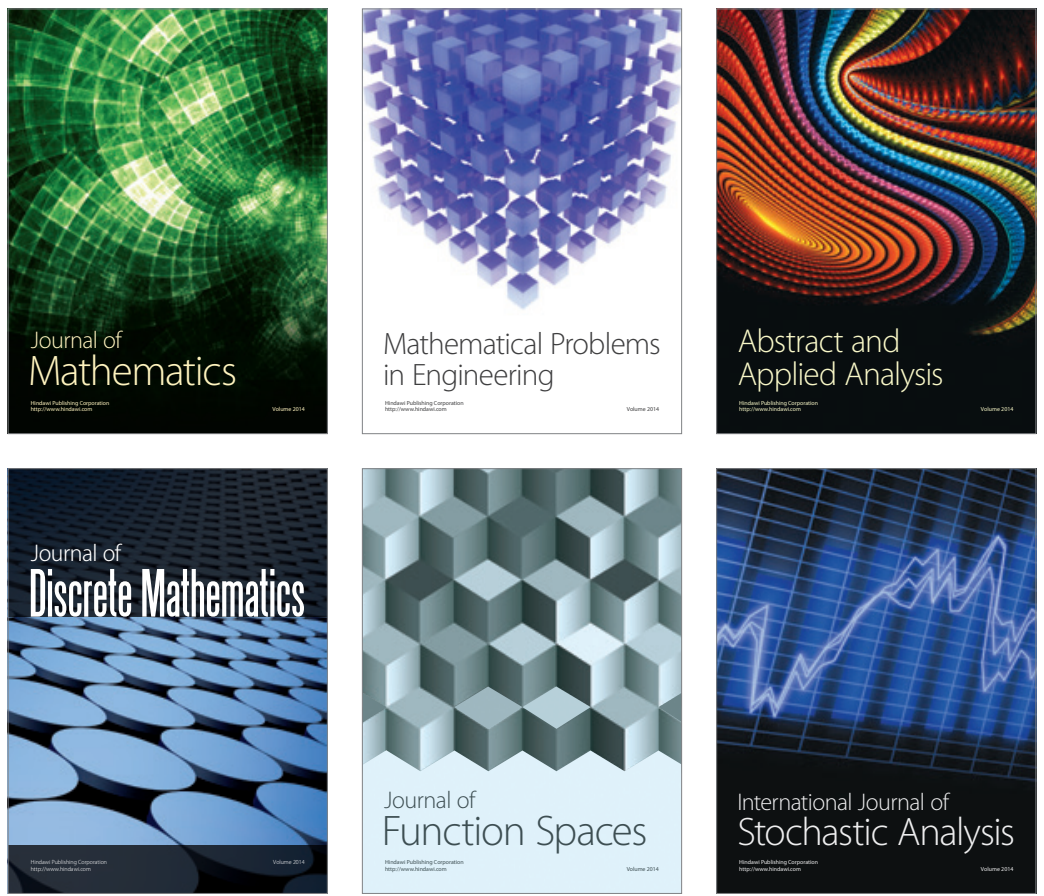

Journal of

Function Spaces

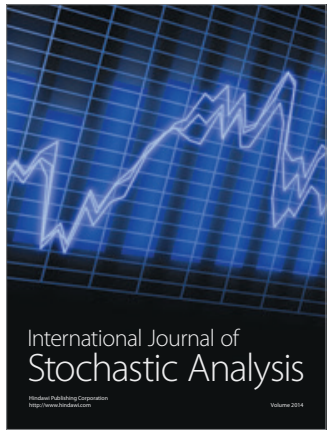

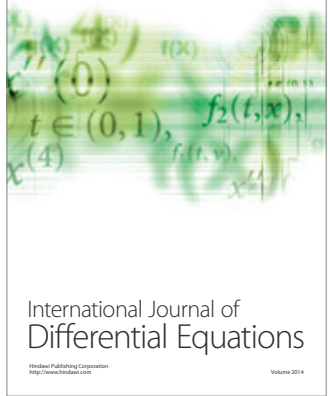
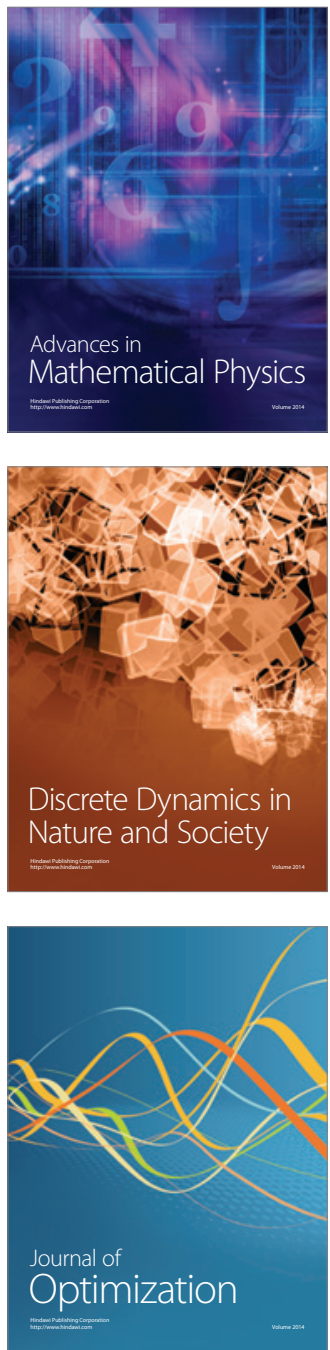